\title{
EXTENDED AORTIC REPLACEMENT FOR ACUTE TYPE A DISSECTION WITH THE TEAR IN THE DESCENDING AORTA
}

Terushisa Kazui, MD

Yukihiko Tamiya, MD

Toshiaki Tanaka, MD

Sakuzo Komatsu, MD
Objective: There has been controversy as to the selection of surgical treatments for acute type $A$ dissection with the tear in the descending thoracic aorta, a subtype of acute aortic dissection in which the limited tear is located distal to the left subclavian artery but the dissection extends retrogradely to the ascending aorta. Methods: Total replacement of the ascending aorta and aortic arch was performed in 12 patients with acute type $A$ dissection with the tear in the descending thoracic aorta between March 1991 and the end of September 1995. The indications for total replacement of the ascending aorta and aortic arch were cardiac tamponade, acute aortic regurgitation, cerebral ischemia, and dilatation of the ascending aorta. The operation was performed with the aid of extracorporeal circulation, blood cardioplegia, selective cerebral perfusion, and open distal anastomosis. The surgical procedure used was total replacement of the ascending aorta and aortic arch with a graft provided with three limbs accompanied by resection of the intimal tear in the descending thoracic aorta. Results: Hospital death occurred in two patients (16.7\%). In both, death was due to dissection/related complications of renal/mesenteric ischemia. The other 10 patients have had uneventful postoperative courses over a mean period of 24 months. Conclusions: Total replacement of the ascending aorta and aortic arch accompanied by resection of an intimal tear distal to the left subclavian artery seems to be justified in selected patients with acute type $A$ dissection with the tear in the descending thoracic aorta. (J Thorac Cardiovasc Surg 1996;112:973-8)
D issecting aneurysm of the thoracic aorta has been classified by DeBakey and associates ${ }^{1}$ and by the Stanford group. ${ }^{2}$ The latter classification has been used more widely in recent years because of its usefulness for evaluation of the prognosis of the disease and determination of the therapeutic approach. It is currently generally accepted that Stanford type A acute aortic dissection and uncomplicated acute type $B$ aortic dissection are treated by emergency surgery and medical treatments, respectively. Moreover, type A aortic dissection requires

From the Department of Thoracic and Cardiovascular Surgery, Sapporo Medical University School of Medicine, Sapporo, Japan.

Received for publication April 11, 1996; revisions requested May 14, 1996; revisions received June 11, 1996; accepted for publication June 13, 1996.

Address for reprints: Teruhisa Kazui, MD, Department of Thoracic and Cardiovascular Surgery, Sapporo Medical University School of Medicine, South 1, West 17, Chuo-ku, Sapporo 060, Japan.

Copyright (C) 1996 by Mosby-Year Book, Inc.

$0022-5223 / 96 \$ 5.00+0 \quad \mathbf{1 2 / 1 / 7 5 8 9 5}$ median sternotomy and total cardiopulmonary bypass, whereas type $\mathrm{B}$ aortic dissection requires left thoracotomy and partial femoro-femoral bypass. However, the existence of a subtype that does not fit this classification and therapeutic guidelines has also been suggested: acute type $A$ dissection with the tear in the descending thoracic aorta, in which the intimal tear is located in the descending thoracic aorta near the left subclavian artery and the dissection extends retrogradely to the ascending aorta. This subtype was termed DeBakey type III-D by Reul and associates, ${ }^{3}$ type III retrograde dissection extending to the aortic arch and ascending aorta by Erbel and coworkers, ${ }^{4}$ descending/type A by Lansman, Ergin, and Griepp, ${ }^{5}$ and non-A/non-B aortic dissection by von Segesser and colleagues. ${ }^{6}$ The prognosis of this subtype is considered to be extremely poor, 3,4 and the selection of therapeutic approaches and surgical procedures has been controversial. We have performed total replacement of the ascending aorta and aortic arch through a median sternotomy in these patients. In this study, 
the indications, techniques, and results of the operation are described.

\section{Patients and methods}

Patients. There were 12 patients with acute aortic dissection, in which an intimal tear was located near the left subclavian artery and the dissection extended retrogradely to the ascending aorta, who underwent total replacement of the ascending aorta and aortic arch between March 1991 and the end of September 1995. During this period, 56 patients with descending thoracic aortic dissection were treated, and the lesions in 16 patients $(29 \%)$ were classified as of this subtype. Four of these patients were excluded from this study: one patient underwent replacement of the descending thoracic aorta through a left thoracotomy because the lesion was located primarily in the descending thoracic aorta, two patients received only medical treatments because of thrombotic occlusion of the false lumen in the ascending aorta, and one patient died of acute myocardial infarction as the dissection extended to the trunk of the left main coronary artery during medical treatments. On the other hand, 60 patients with acute type A aortic dissection underwent the emergency operation, and the lesions in 12 patients (20\%) were confirmed as of this subtype at the time of operation.

The eight male and four female subjects ranged in age from 46 to 75 years, with a mean of $60 \pm 9$ years. Preoperative clinical symptoms, which included precordial pain, back pain, or abdominal pain, were present in all patients. As complications, shock was observed in three patients, cardiac tamponade in six, left pleural rupture in three, acute aortic regurgitation in six, cerebral ischemia in two (one had transient right hemiplegia and the other had loss of consciousness, in which the compromise of arch vessels was confirmed by angiography), and renal/ mesenteric ischemia in two. Four patients required endotracheal intubation and assisted ventilation because of preoperative cardiopulmonary insufficiency. Total replacement of the ascending aorta and aortic arch was indicated for cardiac tamponade in four patients, cardiac tamponade, acute aortic regurgitation, and cerebral ischemia in two, acute aortic regurgitation and cardiac tamponade in two, acute aortic regurgitation in two, and enlargement of the diameter of the ascending aorta $(5 \mathrm{~cm}$ or greater) in two. The time from the onset of dissection to the operation ranged from 6.5 to 76 hours, with a mean of $39.9 \pm 27.5$ hours.

Operative techniques. The heart, ascending aorta, and aortic arch were exposed through a median sternotomy and the arch vessels through bilateral supraclavicular incisions, $5 \mathrm{~cm}$ in length, after partial division of sternocleidomastoid muscles. After heparin was administered, extracorporeal circulation was instituted by draining blood from the right atrium using a single two-staged cannula with return of the blood into the femoral artery. A left ventricular vent was inserted via the right superior pulmonaty vein. Fig. 1 shows the surgical technique. The ascending aorta was crossclamped, the aorta was incised, and blood cardioplegic agent was infused directly into the orifices of the left and right coronary arteries. After the internal surface of the ascending aorta and the aortic valve were examined, the aorta was completely transected immediately above the aortic commissure. Aortic valve resuspension was performed in six patients with acute aortic regurgitation. Then the proximal false lumen was obliterated by applying Teflon felt to the internal and external surfaces of the aorta. In the four patients treated in 1995, gelatin-resorcin-formaldehyde glue ${ }^{7}$ was infused into the proximal false lumen, and Teflon felt was applied only to the external surface of the aorta (Fig. 1, $A$ ). In one patient with coronary artery disease, a bypass operation was performed using a vein graft to the left anterior descending coronary artery. By this time, the patient's rectal temperature was cooled to about $22^{\circ} \mathrm{C}$ by extracorporeal circulation. After circulatory arrest had been instituted, the ascending aorta was unclamped. The innominate artery and left common carotid artery were cannulated from inside the aorta (Fig 1,B). Then selective cerebral perfusion was started at a perfusion rate of 10 $\mathrm{ml} / \mathrm{kg}$ per minute for protection of the brain during reconstruction of the aortic arch. The details regarding selective cerebral perfusion have been described previously. ${ }^{8}$ The internal surface of the aortic arch and descending thoracic aorta was examined during arrest of the circulation via the femoral artery. In all patients an intimal tear was observed in the descending thoracic aorta distal to the origin of the left subclavian artery. After the descending thoracic aorta distal to the intimal tear was completely transected, the distal false lumen was obliterated by the same method as was the proximal false lumen (Fig. 1, B). At this time, the descending thoracic aorta was exposed when necessary by opening the chest through the longitudinal incision of the left parietal pleura. A four-limbed arch graft was prepared by an assistant surgeon during the operation. A woven double velour Hemashield graft (Meadox Medicals, Inc., Oakland, N.J.), 24 to $26 \mathrm{~mm}$ in diameter for the arch graft, was attached with a $12 \mathrm{~mm}$ Hemashield graft for the innominate artery and an $8 \mathrm{~mm}$ Hemashield graft for the left common carotid and left subclavian arteries. The fourth limb, used for antegrade systemic perfusion, was sutured to the side of the arch graft at the same level of the left subclavian artery. Then this graft was sutured to the stump of the descending thoracic aorta with a 3-0 polypropylene running suture (Fig. 1, C). The distance between the origin of the left subclavian artery and the stump of the descending thoracic aorta ranged from 2 to $8 \mathrm{~cm}$, with a mean of $4.5 \pm 1.5$ $\mathrm{cm}$. In the first five patients to be treated, after completion of the distal graft anastomosis, antegrade systemic circulation was started from the side limb of the left subclavian artery graft, and rewarming was begun. Then a proximal graft anastomosis was performed with a 3-0 polypropylene running suture, and coronary perfusion was started. The innominate artery and left common carotid artery were anastomosed to each limb of the graft with 4-0 polypropylene running sutures used in succession. The left subclavian artery was reconstructed after termination of extracorporeal circulation. In the seven patients treated after November 1993, the surgical procedure was changed as described below for protection of the left vertebral artery region. After completion of the distal graft anastomosis, the left subclavian artery was sutured to a side limb of the graft (Fig. 1,D). The proximal end of the graft was 

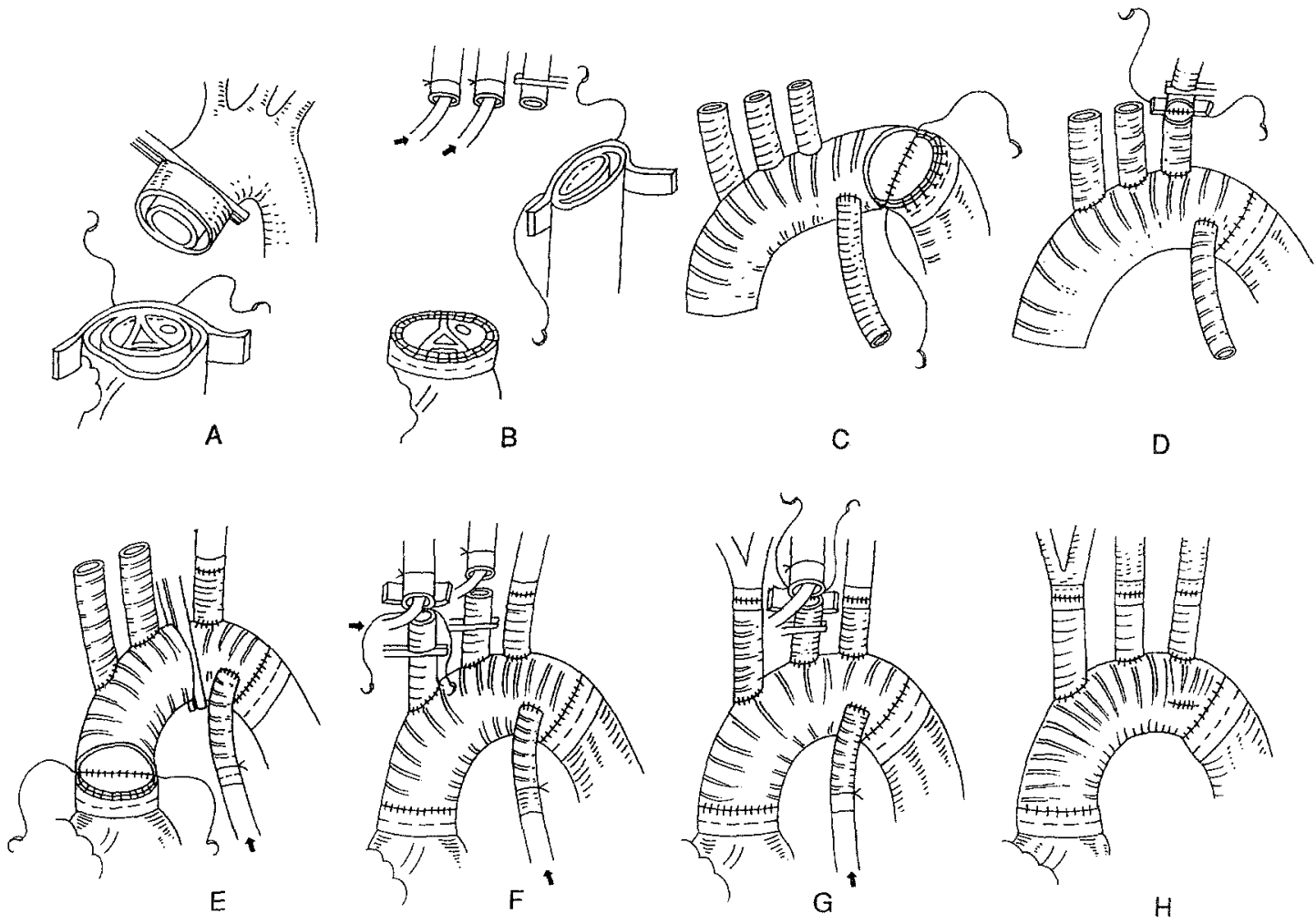

Fig. 1. A scheme of the surgical technique of total replacement of the ascending aorta and aortic arch using a graft provided with branches for acute type A dissection with the tear in the descending thoracic aorta. See the text for details.

crossclamped, antegrade systemic circulation was started via the fourth side limb, and rewarming was begun (Fig. 1, $E$ ). The proximal graft anastomosis was then performed, and coronary perfusion was started. The innominate artery was then anastomosed to the limb of the arch graft with a 4-0 polypropylene running suture (Fig. 1, F). Before completion of the graft anastomosis, the cerebral perfusion cannula was removed. After a $50 \%$ reduction in volume of cerebral perfusion, the left common carotid artery was anastomosed to the limb of the arch graft in a similar fashion (Fig. 1, $G$ ). After termination of extracorporeal circulation, the fourth side branch used for antegrade systemic circulation was removed (Fig. 1, H).

The mean total pump time was $221 \pm 27$ minutes, cardiac ischemic time $129 \pm 28$ minutes, selective cerebral perfusion time $99 \pm 19$ minutes, and open distal anastomosis time $52 \pm 17$ minutes.

\section{Results}

All of the patients regained consciousness after the operation without any distinct evidence of stroke. Two patients who had transient cerebral ischemia before the operation recovered completely. As postoperative complications, pulmonary insufficiency that necessitated assisted ventilation for more than 5 days was observed in five patients, renal insufficiency with a blood urea nitrogen level of more than $70 \mathrm{mg} / \mathrm{dl}$ or a serum creatinine level of more than $3.0 \mathrm{mg} / \mathrm{dl}$ in three patients, and low cardiac output necessitating intraaortic balloon pumping in one patient. Hospital death occurred in two patients $(16.7 \%)$, and death was related to the dissection/related complication of renal/mesenteric ischemia in both patients. One died on the thirteenth postoperative day, and the other died 4 months after the operation of multiple organ failure.

Aortography and computed tomographic scan were performed after the operation in 10 patients to evaluate the state of the false lumen in the distal descending thoracic aorta; closure of the false lumen was observed in five (50\%) (Figs. 2 and 3). The 10 patients excluding the two patients who died in the hospital had uneventful courses and were discharged in good condition. No late death has been observed during a follow-up period of 4 months to 4 years, 11 months (mean $24 \pm 20$ months). Two patients underwent reoperation in the late postop- 

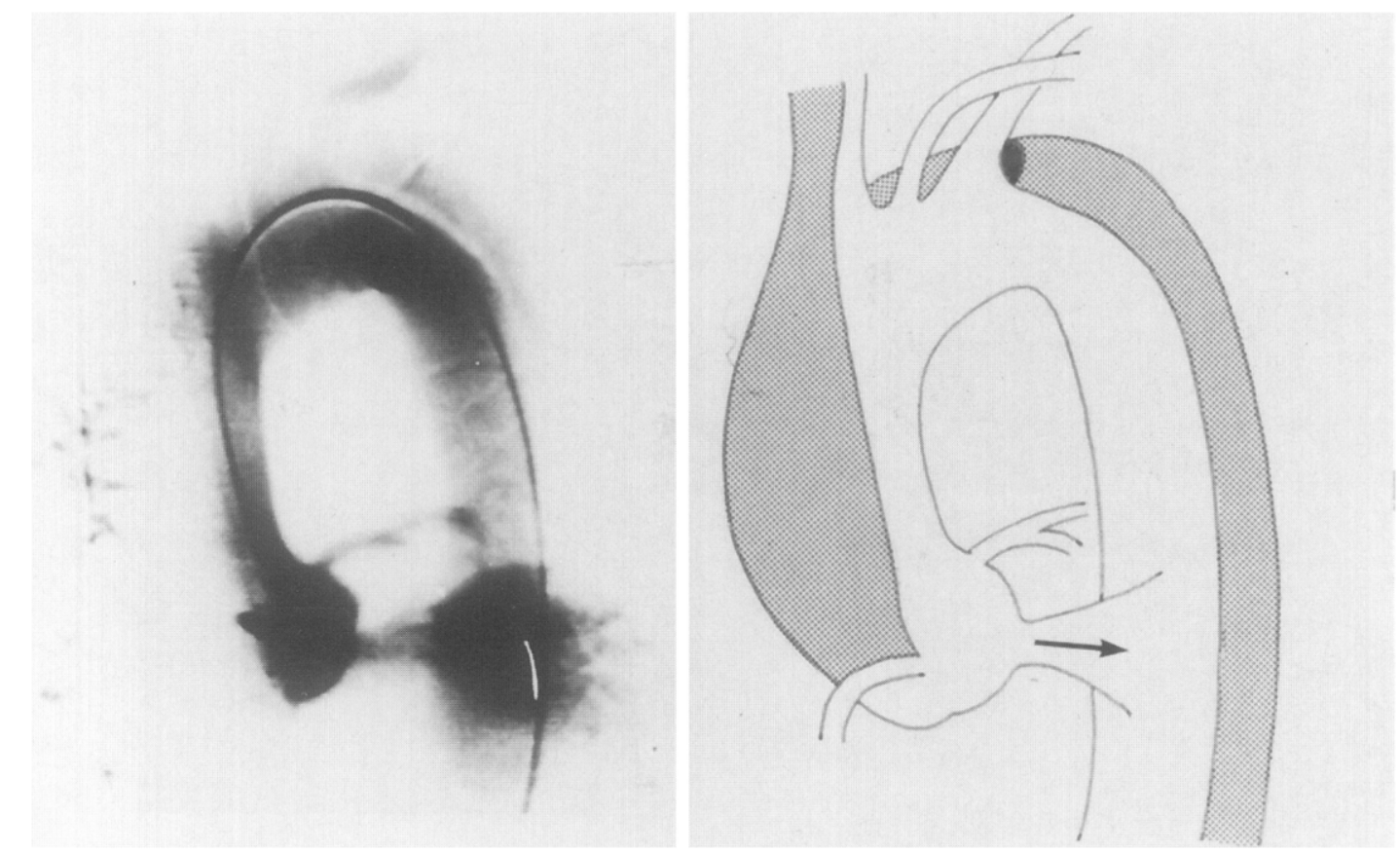

Fig. 2. A preoperative digital subtraction angiographic image in acute type A dissection with the tear in the descending thoracic aorta. The primary intimal tear is located near the left subclavian artery, and the dissection extends retrogradely to the ascending aorta. This patient had acute aortic regurgitation and extension of the dissection to the innominate artery.

erative period. One patient had total replacement of the descending thoracic aorta 4 years, 9 months after the initial operation for aneurysmal formation of the distal false lumen owing to leakage at the distal anastomosis of the graft, and the other had aortoiliac graft replacement 6 months after the initial operation for abdominal aortic aneurysm.

\section{Discussion}

In dissecting aneurysm of the thoracic aorta, the dissection extends not only antegradely but also retrogradely from the site of intimal tear. A typical retrograde dissection is type A dissection with the tear in the descending thoracic aorta, a subtype of aortic dissection in which the intimal tear extends from a site near the left subclavian artery retrogradely to the ascending aorta. Differentiation of this subtype from acute type A dissection, in which the intimal tear is located in the ascending aorta, is not always easy. The precise prevalence of this subtype is not clear, but it is considered by some to occur more than is generally expected. Reul and colleagues $^{3}$ classified nine $(10 \%)$ of 91 cases of
DeBakey type III dissection as this subtype in 1975, and in 1984 Miller and coworkers ${ }^{9}$ classified as this subtype five (10\%) of 48 cases of dissection in which the intimal tear was located in the descending thoracic aorta. In 1993 Erbel and associates ${ }^{4}$ reported that $22(27 \%)$ of 82 cases of DeBakey type III were of this subtype, and in 1994 Lansman and colleagues ${ }^{10}$ reported that five $(7 \%)$ of 69 cases of acute type $A$ dissection treated surgically were of this subtype. In many of our patients, we performed emergency operations under the tentative diagnosis of acute type A dissection without determining the location of the intimal tear because of the urgency of the preoperative condition, only to intraoperatively confirm the presence of an intimal tear near the left subclavian artery and make the diagnosis of this subtype. Therefore it is likely that this subtype was present in many patients heretofore diagnosed with acute type A dissection. At present, in patients with acute type A dissection when the intimal tear is not located in the ascending aorta, the proximal descending thoracic aorta as well as the ascending aorta and aortic arch are usually examined during the operation under circulatory arrest. Therefore 


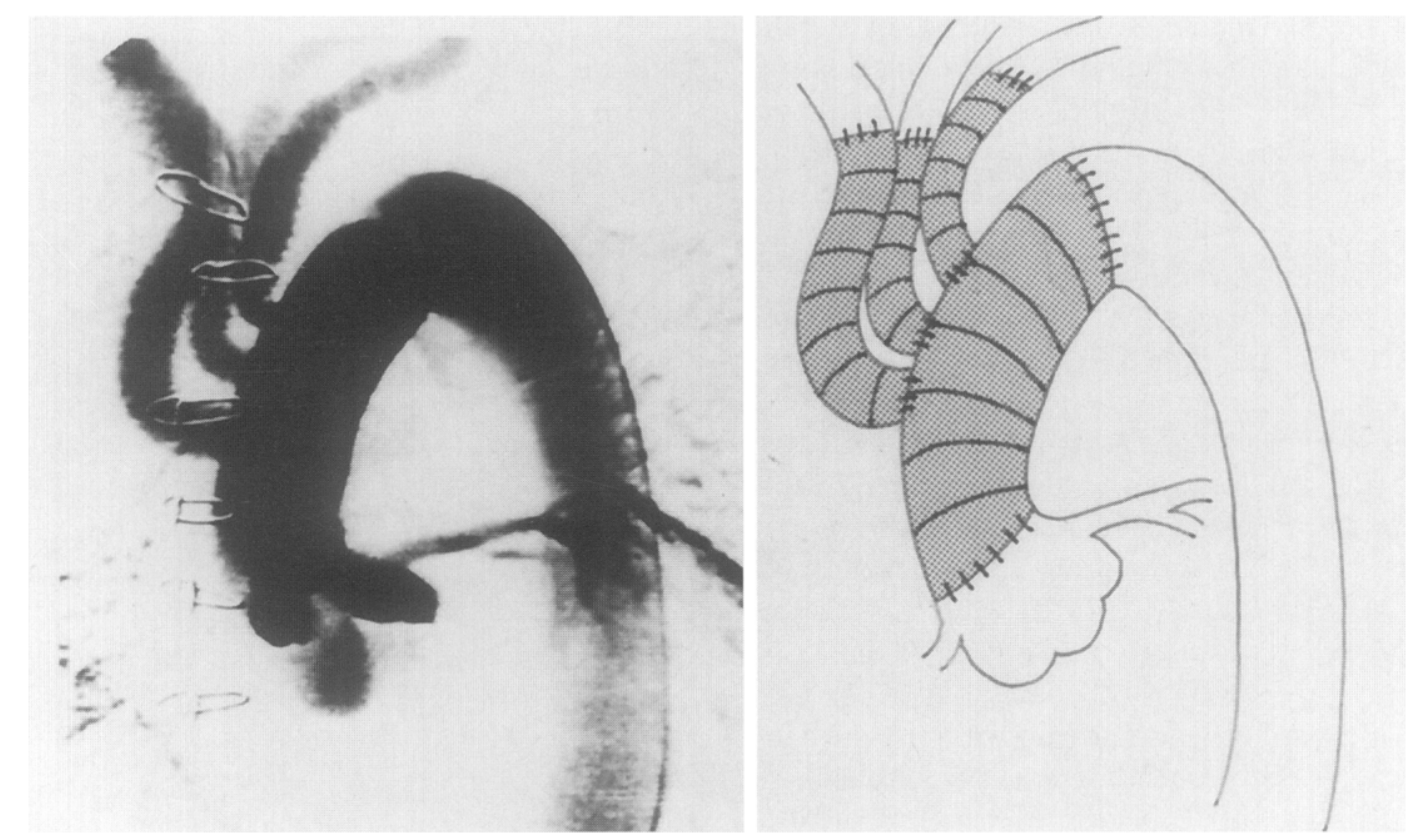

Fig. 3. A postoperative digital subtraction angiographic image in the same patient. The ascending aorta, aortic arch, and arch vessels were reconstructed satisfactorily. The aortic regurgitation has disappeared, and the false lumen of the descending thoracic aorta is occluded.

the diagnostic frequency of this subtype is expected to increase.

The selection of surgical treatments for this subtype has been controversial. ${ }^{3,4,6,9,11}$ In patients in whom the false lumen of the ascending aorta is thrombosed early after the onset and no dilation of the ascending aorta is observed, the treatments are, in principle, the same as those for acute type B aortic dissection. Specifically, replacement of the descending thoracic aorta accompanied by resection of the intimal tear through a left thoracotomy should be performed only for patients with rupture of the descending thoracic aorta and distal organ ischemia, whereas medical treatments are recommended in patients without such severe complications. Emergency operation is necessary when the false lumen of the ascending aorta is patent, and the lesion is classified as acute type A dissection. The selection of the surgical procedure in such patients is controversial. Reul and colleagues ${ }^{3}$ repaired the descending thoracic aorta through a left thoracotomy but reported an $89 \%$ mortality rate owing to cardiac tamponade. Repair of the ascending aorta through a median sternotomy is needed in patients with acute aortic regurgitation, cardiac tamponade, cerebral ischemia, and dilatation of the ascending aorta. In such patients, it is a quandary whether the intimal tear of the descending thoracic aorta should be resected simultaneously with total replacement of the aortic arch. Miller and coworkers ${ }^{9}$ reported that in patients with acute type A aortic dissection, there was no significant difference according to whether the intimal tear was resected or not in terms of either the operative or late survival. However, if the intimal tear is not resected, the potential for immediate bleeding from the anastomosis sites, rupture of the false lumen, secondary development of aneurysm from the false lumen in the aortic arch, and reoperation or death owing to rupture might increase. Erbel and coworkers ${ }^{4}$ reported that thrombotic occlusion of the false lumen could not be expected simply by replacement of the ascending aorta in such cases, and that the results such an approach were therefore unsatisfactory. Recently, reconstruction of the aortic arch has been performed more aggressively for acute type A aortic dissection because of marked improvements in the methods of preventing cerebral ischemia during the aortic arch repair. ${ }^{12-15}$ Resection of the intimal tear near the left subclavian artery followed by extended reconstruction by total replacement of the ascending aorta and aortic arch may be the best treatment for this subtype. This extended reconstruction can be performed with ac- 
ceptable mortality and morbidity by the use of a graft provided with three limbs for replacement of the aortic arch, selective cerebral perfusion for protection of the brain, and open distal anastomosis. ${ }^{15}$ Hospital death was observed in two $(16.7 \%)$ of our patients, and the cause of death in both patients was related to renal/ mesenteric ischemia, which is a major risk factor that affects the surgical results. ${ }^{9}$

Access to the point in the descending thoracic aorta distal to the origin of the left subclavian artery by median sternotomy is a problem. In our patients, the graft was anastomosed to the descending thoracic aorta 2 to $8 \mathrm{~cm}$ (mean $4.5 \mathrm{~cm}$ ) from the origin of the left subclavian artery origin after resection of the intimal tear. Usually, the descending thoracic aorta 4 to $5 \mathrm{~cm}$ distal to the origin of the left subclavian artery can be reached without left thoracotomy. In a patient who had rupture into the left thoracic cavity, the descending aorta $8 \mathrm{~cm}$ distal to the origin of the left subclavian arteries could be reached by displacement of the heart and the lung to the right and opening of the thoracic cavity by longitudinal incision of the left pleura.

In one patient, an aneurysm developed from the false lumen because of leakage from the site of the distal aortic anastomosis in the late postoperative period, and reconstruction of the descending thoracic aorta was required. The prevalence of leakage through the needle holes at the site of aortic anastomosis has decreased recently because of the use of gelatin-resorcin-formaldehyde glue and, more recently, because of our use of a modified "elephant trunk" technique in which another smaller-caliber graft is inserted into the true lumen of the descending thoracic aorta. Teflon felt is placed outside the aorta, and then the false lumen is obliterated in a sandwich fashion. The occlusion rate of the false lumen distal to the site of the aortic anastomosis is expected to increase.

In conclusion, extended aortic reconstruction by resection of the intimal tear in the descending thoracic aorta and total replacement of the ascending aorta and aortic arch is considered to be a reasonable surgical technique for the treatment of acute type A dissection with the tear in the descending thoracic aortia complicated by cardiac tampon- ade, acute aortic regurgitation, cerebral ischemia, and dilatation of the ascending aorta.

\section{REFERENCES}

1. DeBakey ME, Henly WS, Cooley DA, Morris GC, Crawford ES, Beall AC Jr. Surgical management of dissecting aneurysm of the aorta. J Thorac Cardiovasc Surg 1965;49: 130-49.

2. Daily PO, Trueblood HW, Stinson EB, Wuerflein RD, Shumway NE. Management of acute aortic dissection. Ann Thorac Surg 1970;10:237-47.

3. Reul GJ Jr, Cooley DA, Hallman GL, Reddy SB, Kyger ER III, Wukasch DC. Dissecting aneurysm of the descending aorta. Arch Surg 1975;110:632-40.

4. Erbel R, Oelert IT, Meyer J, et al. Effect of medical and surgical therapy on aortic dissection evaluated by transesophageal echocardiography: implications for prognosis and therapy. Circulation 1993;87:1604-15.

5. Lansman SL, Ergin MA, Griepp RB. Treatment of acute aortic arch dissection. Ann Thorac Surg 1993;55:816-7.

6. von Segesser LK, Killer I, Ziswiler M, et al. Dissection of the descending thoracic aorta extending into the ascending aorta: a therapeutic challenge. J Thorac Cardiovasc Surg 1994;108: 755-61.

7. Bachet J, Goudot B, Teodori G, et al. Surgery of Type A acute aortic dissection with Gelatine-Resorcine-Formol biological glue: a twelve-year experience. J Cardiovasc Surg 1990;31:263-73.

8. Kazui T, Inoue N, Yamada O, Komatsu S. Selective cerebral perfusion during operation for aneurysm of the aortic arch: a reassessment. Ann Thorac Surg 1992;53:109-14.

9. Miller DC, Mitchell RS, Oyer PE, Stinson EB, Jamieson SW, Shumway NE. Independent determinants of operative mortality for patients with aortic dissections. Circulation 1984; 70(Pt 2):1153-64.

10. Lansman SL, Gall JD, Schor JS, et al. Subtypes of acute aortic dissection. J Card Surg 1994;9:729-33.

11. Cipriano PR, Griepp RB. Acute retrograde dissection of the ascending thoracic aorta. Am J Cardiology 1979;43: 520-8.

12. Massimo CG, Presenti LF, Marranci P, et al. Extended and total aortic resection in the surgical treatment of acute type $A$ aortic dissection: experience with 54 patients. Ann Thorac Surg 1988;46:420-4.

13. Bachet J, Teodori G, Goudot B, et al. Replacement of the transverse aortic arch during emergency for type A aortic dissection: report of 26 cases. J Thorac Cardiovasc Surg 1988;96:878-86.

14. Lansman SL, Raissi S, Ergin MA, Griepp RB. Urgent operation for acute transverse aortic arch dissection. J Thorac Cardiovase Surg 1989;97:334-41.

15. Kazui T, Kimura N, Yamada O, Komatsu S. Total arch graft replacement in patient with acute type A aortic dissection. Ann Thorac Surg 1994;58:1462-8. 